PROCEEDINGS OF THE

AMERICAN MATHEMATICAL SOCIETY

Volume 129, Number 6, Pages 1601-1608

S 0002-9939(00)05702-6

Article electronically published on October 31, 2000

\title{
RIGID CHAINS ADMITTING MANY EMBEDDINGS
}

\author{
M. DROSTE AND J. K. TRUSS
}

(Communicated by Alan Dow)

\begin{abstract}
A chain (linearly ordered set) is rigid if it has no non-trivial automorphisms. The construction of dense rigid chains was carried out by Dushnik and Miller for subsets of $\mathbb{R}$, and there is a rather different construction of dense rigid chains of cardinality $\kappa$, an uncountable regular cardinal, using stationary sets as 'codes', which was adapted by Droste to show the existence of rigid measurable spaces. Here we examine the possibility that, nevertheless, there could be many order-embeddings of the chain, in the sense that the whole chain can be embedded into any interval. In the case of subsets of $\mathbb{R}$, an argument involving Baire category is used to modify the original one. For uncountable regular cardinals, a more complicated version of the corresponding argument is used, in which the stationary sets are replaced by sequences of stationary sets, and the chain is built up using a tree. The construction is also adapted to the case of singular cardinals.
\end{abstract}

\section{INTRODUCTION}

Various authors have constructed algebraic structures having prescribed endomorphism monoids (including the case of small automorphism groups); see, for instance, 44 for a survey on this problem for modules and fields. In 3, strict partial orders with large endomorphism monoid but small automorphism groups were used to solve a problem from universal algebra on Krasner clones. In this paper we wish to construct chains, that is, linearly ordered sets, which have trivial automorphism group but large endomorphism monoid.

Let us say that a structure is rigid if it has no non-trivial automorphisms. Clearly, any ordinal is a rigid chain. However, for any infinite ordinal $\alpha$ there are nonidentity embeddings of $\alpha$ into $\alpha$, that is, order-preserving (and hence one-to-one) maps from $\alpha$ into $\alpha$. Since $\alpha$ is rigid these will necessarily not be onto. There will, however, be a very large number of them, $2^{|\alpha|}$ in fact, where $|\alpha|$ is the cardinality of $\alpha$.

There is another sense in which we could demand that a chain $(X,<)$ admitted 'many' embeddings, namely, that any finite partial automorphism of $(X,<)$ extends to an embedding. (A partial automorphism of $(X,<)$ is an order-isomorphism from a subset of $X$ into $X$.) This requirement does not (ostensibly) imply that there are $2^{|X|}$ embeddings, but it does imply, as is easy to see, that $(X,<)$ is dense linearly ordered without endpoints (provided $|X|>1$ ). This is the sense in which we shall

Received by the editors July 7, 1999 and, in revised form, September 15, 1999.

2000 Mathematics Subject Classification. Primary 06A05.

Key words and phrases. Chain, linearly ordered set, rigid, embedding, meagre, stationary.

Research supported by a grant from the British-German Academic Collaboration Programme. 
use the expression 'admits many embeddings', and our main task will be to show how two methods for constructing rigid chains, ([5] and [1]) can be adapted to give rigid chains admitting many embeddings. We remark at once that since any countable dense chain without endpoints is isomorphic to the chain $\mathbb{Q}$ of rational numbers, which is certainly not rigid, our constructions can only be of uncountable chains.

For comparison we recall that a chain $(X,<)$ is called homogeneous if any finite partial automorphism of $(X,<)$ extends to an automorphism (rather than just an embedding) of $(X,<)$. A homogeneous chain necessarily has a large and rich automorphism group, and such chains and their groups have been extensively studied; for example, see [6].

The first classical construction of a dense rigid chain is due to Dushnik and Miller [5] (see also [7] p. 147). Here the chain is a subset of $\mathbb{R}$, under the usual ordering, and the method of construction is simply to enumerate all possible nonidentity automorphisms, and destroy each successively by transfinite induction. The same construction can be used to destroy even all non-identity order-embeddings of the chain into itself. By contrast, we show here how to adapt this to give such a chain admitting many embeddings by using suitable Cantor sets into which intervals of $\mathbb{R}$ can be embedded, and a Baire category argument. In fact, the chain we construct has the apparently stronger property of being super-dense, by which we mean that the whole chain can be embedded into any of its intervals (and this property clearly implies that the chain admits many embeddings). We remark that it is easy to see that, for a chain of countable coterminality, superdensity is equivalent to saying that each finite partial isomorphism extends to an order-embedding (by use of 'patching'). In particular, any homogeneous chain of countable coterminality is super-dense. The long rational and real lines $\left(\omega_{1}\right.$ copies of $\mathbb{Q}$, and its order-completion, respectively) are, however, homogeneous but not super-dense.

Theorem 1.1. There is a super-dense rigid subset of $\mathbb{R}$ of cardinality $2^{\aleph_{0}}$.

The second construction applies to an arbitrary uncountable cardinal $\kappa$, and is more subtle in the sense that instead of crudely enumerating, and successively destroying, unwanted automorphisms, all members of a dense set of points of the final structure are 'encoded' by certain subsets of the chain, in such a way that automorphisms (but not embeddings) preserve codes. This is more plausible as a general method, since the number of tasks to be performed is equal to $\kappa$. A method of destroying possible automorphisms could apparently involve more tasks. (For $\mathbb{R}$ the whole point is that there are only $2^{\aleph_{0}}$ possible automorphisms.) Since all points in the dense subset have different codes, it follows that the chain is rigid. The extra work here over [1] is to ensure that there are enough embeddings. The proof begins with the case $\kappa$ regular, and is then extended to an arbitrary uncountable cardinal.

Theorem 1.2. For any uncountable cardinal $\kappa$ there is a super-dense rigid chain of cardinality $\kappa$.

We remark that the chains constructed here may be used to provide even sharper solutions to the problem considered in [3], and in $\S 4$ we also note how we can construct chains that are not rigid, but that become rigid on addition, or deletion, of a single point. 


\section{Subchains of the Real Line}

In this section we give the proof of Theorem 1.1. For this we modify the classical construction of Dushnik and Miller [5] so as to give a rigid subchain of $\mathbb{R}$ admitting many embeddings. The method makes rather blatant use of the axiom of choice. (The axiom of choice is also used in $\S 3$ since, for instance, 8 required AC, but its use there is less explicit.) Let us write $\mathfrak{c}$ for the cardinal (initial ordinal) of $\mathbb{R}$, $\mathfrak{c}=2^{\aleph_{0}}$. Since any order-automorphism of $\mathbb{R}$ is continuous, it is determined by its action on $\mathbb{Q}$, so there are $\left(2^{\aleph_{0}}\right)^{\aleph_{0}}=2^{\aleph_{0}}=\mathfrak{c}$ non-trivial order-automorphisms of $\mathbb{R}$. Let them be enumerated as $\left\{f_{\alpha}: \alpha<\mathfrak{c}\right\}$.

Recall that the 'Cantor ternary set' is the subset of the unit interval $[0,1]$ consisting of all points having a ternary expansion in which 1 does not appear. This is an uncountable nowhere dense set, and it has a subset which is order-isomorphic to $\mathbb{R}$. If we remove the lefthand endpoint 0 and all its members having a ternary expansion with eventually all $2 \mathrm{~s}$, what remains is order-isomorphic to the real open interval $(0,1)$, and hence to $\mathbb{R}$. In fact, an order-isomorphism is found by replacing all $2 \mathrm{~s}$ in the ternary expansions by 1 s and re-interpreting the result as a binary expansion. Using this observation, we choose a nowhere dense subset $Y_{I}$ of each open interval $I$ with rational endpoints, which is order-isomorphic to $\mathbb{R}$. Then $Y=\bigcup\left\{Y_{I}: I\right.$ an open interval with rational endpoints $\}$ is meagre (a countable union of nowhere dense sets) and dense, since it intersects every open rational interval.

The construction of our rigid subchain of $\mathbb{R}$ is as follows. Let us choose $x_{\alpha}, y_{\alpha} \in \mathbb{R}$ by transfinite induction. Suppose inductively that $x_{\beta}$ and $y_{\beta}$ have been chosen for all $\beta<\alpha$, where $\alpha<\mathfrak{c}$. Since $f_{\alpha} \neq i d$, there is a non-empty open interval $I_{\alpha}$ with rational endpoints such that $I_{\alpha} \cap f_{\alpha} I_{\alpha}=\emptyset$. Now $I_{\alpha}-f_{\alpha}^{-1} Y$ is comeagre in $I_{\alpha}$, hence of cardinality $\mathfrak{c}$, so there is $x_{\alpha}$ in this set not equal to $x_{\beta}, y_{\beta}, f_{\alpha}^{-1} x_{\beta}$ for $\beta<\alpha$. Let $y_{\alpha}=f_{\alpha} x_{\alpha}$ and $X=\left\{x_{\alpha}: \alpha<\mathfrak{c}\right\} \cup Y$. Then $X$ is the desired subset of $\mathbb{R}$. The intuition is that $\left\{x_{\alpha}: \alpha<\mathfrak{c}\right\}$ and $\left\{y_{\alpha}: \alpha<\mathfrak{c}\right\}$ enumerate the points that we do and do not want to be in $X$ respectively. Any order-automorphism of $\mathbb{R}$ will take $x_{\alpha}$ to $y_{\alpha}$ for some $\alpha$, so does not induce an automorphism of $X$.

To make this more precise, we first observe that all the $x_{\alpha}$ are distinct, and are also different from all the $y_{\alpha}$. For $x_{\alpha}$ was explicitly chosen different from all earlier $x_{\beta}$ and $y_{\beta}$, and as $x_{\alpha} \neq f_{\alpha}^{-1} x_{\beta}$ for $\beta<\alpha$, and $y_{\alpha}=f_{\alpha} x_{\alpha}$, also $y_{\alpha} \neq x_{\beta}$. Since $x_{\alpha} \in I_{\alpha}, y_{\alpha} \in f_{\alpha} I_{\alpha}$, and these two intervals are disjoint, $x_{\alpha} \neq y_{\alpha}$. Furthermore, as $x_{\alpha} \notin f_{\alpha}^{-1} Y, y_{\alpha} \notin Y$. Now, since $Y$ is dense, and is contained in $X$, any nontrivial order-automorphism of $X$ induces an order-automorphism of $\mathbb{R}$, so is the restriction to $X$ of some $f_{\alpha}$. But $x_{\alpha} \in X$ and $f_{\alpha} x_{\alpha}=y_{\alpha} \notin X$, so that $f_{\alpha}$ is not an automorphism of $X$ after all.

Finally, we show that $(X,<)$ is super-dense (from which, as we remarked, the fact that it admits many embeddings is immediate) as follows. Let $x<y$ in $X$. Then there is a non-empty open interval $I$ with rational endpoints contained in $(x, y)$, and $(\mathbb{R},<)$ is isomorphic to $Y_{I} \subseteq X$. This isomorphism restricts to an embedding of $X$ into $X \cap(x, y)$, as desired.

\section{Chains of Cardinality $\kappa$}

We now move on to the proof of Theorem [1.2, In [1] rigid chains having various additional properties were constructed. We use here essentially the same method (somewhat simplified), modifying the argument so that there will also be 'many embeddings' of the resulting chains. We begin by supposing that $\kappa$ is an uncountable 
regular cardinal, and show at the end how to relax the regularity hypothesis. As in [1] we appeal to Solovay's result [8], that any stationary subset of an uncountable regular cardinal $\kappa$ can be expressed as the disjoint union of $\kappa$ pairwise disjoint stationary sets. (We recall that a subset of $\kappa$ is said to be stationary if it intersects each closed unbounded subset of $\kappa$, where 'closed' here means with respect to the order topology.)

The 'basic construction' of a rigid chain is as follows. We let $\mathbb{L}_{\kappa}$ be the 'long rational line' of length $\kappa$, that is, $\kappa \cdot \mathbb{Q}$ under the lexicographic ordering, $(\kappa$ copies of $\mathbb{Q}$ ). As a variant of this, for any set $S$ of non-zero ordinals, we let $\mathbb{L}_{\kappa, S}$ be $\kappa . \mathbb{Q} \cup\{(\alpha,-\infty): \alpha \in S\}$, again ordered lexicographically. This is the same as $\mathbb{L}_{\kappa}$ except that a point $(-\infty)$ has been inserted in the gaps between the copies of $\mathbb{Q}$ corresponding to elements of $S$. They are added on the left $(-\infty$ rather than $\infty)$ so that there is continuity at limit points of $S$ (if $\lambda$ is a limit point of $S$, then $(\lambda,-\infty)$ is the limit of $\{(\alpha,-\infty): \alpha<\lambda, \alpha \in S\})$. We exclude 0 from $S$ because we want the chains not to have left-hand endpoints.

Now let $\mathcal{A}$ be a family of $\kappa$ pairwise disjoint stationary subsets of $\kappa-\{0\}$, and we enumerate the members of $\mathcal{A}$ in $\omega$ disjoint sets $\mathcal{A}_{n}$ of size $\kappa$. The members of $\mathcal{A}_{n}$ are the stationary sets which are used at the $n$th stage of the construction as 'codes'. Define a sequence of chains $\left\{\left(X_{n},<\right): n \in \omega\right\}$ by induction. At each stage we shall adjoin copies of $\mathbb{L}_{\kappa, S}$ for various $S$, and inside these we have a clear notion of the 'copies' of $\mathbb{Q}$ added at that stage (that is, the points in $\mathbb{L}_{\kappa}$ ).

Let $X_{0}=\mathbb{L}_{\kappa}$. Assuming that $X_{n}$ has been chosen, we let $X_{n+1}$ be formed by adjoining to $X_{n}$ sets of the form $\mathbb{L}_{\kappa, S_{x}}$ immediately to the left of each $x$ which in $X_{n}$ lies in a copy of $\mathbb{Q}$ added at the previous stage, where the $S_{x}$ are distinct members of $\mathcal{A}_{n}$. More precisely, this means that for any $y<x$ in $X_{n}$, and $z$ lying in this copy of $\mathbb{L}_{\kappa, S_{x}}, y<z<x$. We may say that the points of $X_{n}$ lying in a copy of $\mathbb{Q}$ that has been adjoined at the previous stage 'require codes', and once the $\mathbb{L}_{\kappa, S_{x}}$ has been added immediately to the left of $x$, that $x$ 'has been coded'. The idea is that $S_{x}$ should be the code of $x$, but this only makes any sense if we can somehow recognize $S_{x}$ intrinsically from the chain $X_{n+1}$ (and even in the limit $X=\bigcup_{n \in \omega} X_{n}$, so that, once a point has been coded, it retains that code at all subsequent stages). At any rate, all points of $X_{n}$ requiring codes are limits of an increasing sequence of order-type $\omega$ (we say they have cofinality $\omega$ ), but after being encoded, they have cofinality $\kappa$, and the set of points of $X$ that have been coded by stage $n+1$ is dense in $X_{n}$.

The chain constructed by the above method is then $X=\bigcup_{n \in \omega} X_{n}$. It is clear that it has cardinality $\kappa$. Since each $X_{n}$ is densely ordered without endpoints, the same is true of $X$. The main point is to show why it is rigid. Suppose that $f$ is a non-trivial automorphism of $(X,<)$. Since the set of coded points of $X$ is dense, some coded point $x$ is moved by $f$, to $y$ say. Now let us say that a Dedekind cut of a linearly ordered set is a pair of disjoint non-empty subsets with union the whole set, one closed downwards (the lower cut), one closed upwards (the upper cut). A point in a linear ordering extending the original is said to realize the cut if it lies strictly above all the points in the lower cut and strictly below all the points in the upper cut. Now the only Dedekind cuts of any $X_{n}$ that are realized during the construction are those of the form $(\{z: z<x\},\{z: x \leq z\})$ for $x \in X_{n}$. Moreover, such cuts are only realized at one stage (in the sense that the similarly defined cut ( $\{z: z<x\},\{z: x \leq z\})$ of $X_{n^{\prime}}$ for $n^{\prime}>n$ is never realized). It follows that $x$ is the supremum of a set of type $S_{x}$ in $X$ (namely, the copy of $S_{x}$ that was added 
when it was coded). Moreover, if we write $L_{x}$ for the subset of the order-completion of $\mathbb{L}_{\kappa, S_{x}}$ consisting of $\{(\alpha,-\infty): \alpha \in \kappa-\{0\}\}$, where $n$ is the stage where $x$ was coded, then $L_{x}$ is a closed unbounded subset of $(-\infty, x) \cap \overline{X_{n+1}}$, (where $\overline{X_{n+1}}$ is the closure of $X_{n+1}$ in $X$ under the order topology), and since none of the cuts $(\{z: z<t\},\{z: t \leq z\})$ for $t \in L_{x}$ is realized at any stage, $L_{x}$ is still closed unbounded in $X$. In addition, $L_{x} \cap X$ is still the same copy of $S_{x}$ that was added at stage $n$. Similar remarks apply to $S_{y}$ and $L_{y}$.

Let $L_{x}=\left\{x_{\alpha}: 0<\alpha<\kappa\right\}$ and $L_{y}=\left\{y_{\alpha}: 0<\alpha<\kappa\right\}$ be the increasing enumerations of these two sets. Since $(X,<)$ is densely ordered, $f$ extends to an order-automorphism of its order-completion. Standard arguments involving closed unbounded subsets of uncountable cardinals show that $C=\left\{\alpha: f x_{\alpha}=y_{\alpha}\right\}$ is a closed unbounded subset of $\kappa$. Since $S_{x}$ is stationary, $S_{x} \cap C \neq \emptyset$. Let $\alpha \in S_{x} \cap C$. Then $x_{\alpha} \in X$, so $y_{\alpha}=f x_{\alpha} \in X$. Hence $\alpha \in S_{y}$, contrary to $S_{x} \cap S_{y}=\emptyset$. This contradiction shows that $(X,<)$ is rigid.

To obtain a rigid chain admitting many embeddings we need to iterate the above construction. To start with, we work with finite sequences of stationary sets, rather than individual stationary sets. The other new feature needed here is that we shall want to realize cuts other than the ones of the form $(\{y: y<x\},\{y: x \leq y\})$. These are needed to fulfill a role similar to the set $Y$ in the previous section, which 'absorbed' lots of copies of open intervals, even though it was itself 'small' (there, meagre). Since we must, at all costs, avoid realizing cuts that will destroy the coding procedure, we choose these cuts to be irrational ones (meaning that the lower cut has no greatest element, and the upper cut no least). Since we are constantly adjoining copies of $\mathbb{Q}$, it is clear what this means, and also that there is an abundance of such cuts (in particular, they are dense).

This time, let $\mathcal{A}$ be a family of $\kappa$ finite non-empty sequences of stationary subsets of $\kappa-\{0\}$, such that any two final entries of the sequences are disjoint. Once more, we subdivide $\mathcal{A}$ into $\omega$ disjoint sets $\mathcal{A}_{n}$ of size $\kappa$. If $\underline{S}=\left(S_{0}, S_{1}, \ldots, S_{m-1}\right)$ we let $\mathbb{L}_{\kappa, \underline{S}}=\mathbb{L}_{\kappa, S_{0}} \wedge \mathbb{L}_{\kappa, S_{1}} \wedge \ldots^{\wedge} \mathbb{L}_{\kappa, S_{m-1}}$ where ${ }^{\wedge}$ denotes 'concatenation', the sum of disjoint copies of the $\mathbb{L}_{\kappa, S_{i}}$. That is, the domain of $\mathbb{L}_{\kappa, \underline{S}}$ may be taken to be

$$
m \times \kappa \times \mathbb{Q} \cup \bigcup\left\{\{i\} \times S_{i} \times\{-\infty\}: i<m\right\}
$$

where

$$
\begin{aligned}
(i, \alpha, q)<(j, \beta, r) & \text { if } \quad i<j, \\
& \text { or } \quad i=j \wedge \alpha<\beta, \\
& \text { or } \quad i=j \wedge \alpha=\beta \wedge q<r .
\end{aligned}
$$

Let $X_{0}=\mathbb{L}_{\kappa}=\kappa \cdot \mathbb{Q}$. Assuming that $X_{n}$ has been defined, we adjoin, immediately to the left of each point $x$ of $X_{n}$ lying in a copy of $\mathbb{Q}$, a copy of $\mathbb{L}_{\kappa, \underline{S}_{r}}$, where $\underline{S}_{x}$ are distinct members of $\mathcal{A}_{n}$, to form $X_{n+1}$. Again, we say that such $x$ has been coded.

Let $X=X_{\mathcal{A}}=\bigcup_{n \in \omega} X_{n}$. Then the previous proof that $X$ is rigid carries through to this version. The point is that it is really only the last entry of each sequence that is significant for the coding, and we demanded that these were all distinct. So this construction appears as a somewhat minor variant of the previous one. The point is that there is a chain $Y$ resulting from the construction performed with subsequences of the members of $\mathcal{A}$ which can now be embedded in $X$. More precisely, if $\mathcal{A}$ and $\mathcal{B}$ are families of finite sequences of stationary sets as above, and $\mathcal{A}$ is obtained from $\mathcal{B}$ by deleting all final entries, then $X_{\mathcal{B}}$ has a subset of the form 
$X_{\mathcal{A}}$, which we get by deleting the final copies of $\kappa \cdot \mathbb{Q}$, and any elements 'depending on' these, and any duplicates. (Note that $\mathcal{A}$ does not itself totally determine $X_{\mathcal{A}}$, but we also need to know the manner in which the sequences of stationary sets are assigned during the construction. For this reason we talk of a chain as having the 'form' $X_{\mathcal{A}}$, to avoid implying that it is unique.) Conversely, if we start with any chain $Y$ of the form $X_{\mathcal{A}}$, we can construct a chain $X$ of the form $X_{\mathcal{B}}$ such that $Y$ can be embedded in $X$.

We are now ready to perform the construction. Let $I_{0} \subset I_{1} \subset I_{2} \subset \ldots$ be index sets of cardinality $\kappa$ such that $\left|I_{j+1}-I_{j}\right|=\kappa$ for each $j$, and let $\Sigma$ be the family of all finite sequences $\left(i_{0}, i_{1}, \ldots, i_{n-1}\right)$ such that for each $j, i_{j} \in I_{j}$ (including the empty sequence). Let $\mathcal{A}$ be a family of $\kappa$ pairwise disjoint stationary subsets of $\kappa-\{0\}$. Since $|\kappa \times \Sigma|=\kappa$ we may let

$$
\mathcal{A}=\left\{S_{\alpha \sigma}: \alpha \in \kappa, \sigma \in \Sigma\right\} .
$$

Let

$$
X_{\sigma}=X_{\left\{\left(S_{\alpha()}, S_{\alpha(\sigma(0))}, S_{\alpha(\sigma(0) \sigma(1))}, \ldots, S_{\alpha \sigma}\right): \alpha<\kappa\right\}}
$$

where $\sigma \in \Sigma$, as defined above by the 'general method' for constructing rigid chains. By the previous paragraph, we may suppose that $X_{\sigma}$ embeds in $X_{\sigma^{\wedge}(i)}$ for each $i$, and we choose such an embedding $f_{\sigma, i}$. By composing these maps we may find embeddings $f_{\sigma, \tau}$ of $X_{\sigma}$ into $X_{\sigma^{\wedge} \tau}$.

Choose a dense set $T_{\sigma}=\left\{t_{\sigma}^{i}: i \in I_{n}\right\}$ of $\kappa$ irrational cuts of $X_{\sigma}$ for $\sigma$ of length $n$, so that for each $\sigma$ and $i, f_{\sigma, i} T_{\sigma} \subseteq T_{\sigma^{\wedge}(i)}$. This is possible, since under $f_{\sigma, i}$, irrational cuts are mapped to irrational cuts. We suppose that the enumerations are chosen so that $f_{\sigma, i} t_{\sigma}^{i^{\prime}}=t_{\sigma^{\wedge}(i)}^{i^{\prime}}$, which we can do by suitably enumerating $T_{\sigma^{\wedge}(i)}$, remembering that we have $\kappa$ 'spare' points in $I_{n+1}$ to use for the members of $T_{\sigma^{\wedge}(i)}-f_{\sigma, i} T_{\sigma}$. For the composed embeddings, we deduce that $f_{\sigma, \tau} t_{\sigma}^{i^{\prime}}=t_{\sigma^{\wedge} \tau}^{i^{\prime}}$.

The chain $Y$ that we construct is taken to be the disjoint union of the $X_{\sigma}$ for $\sigma \in \Sigma$. Since each $X_{\sigma}$ is itself a chain, to specify the ordering on $Y$ we just have to say how elements of distinct $X_{\sigma}$ s are related, and this is where the cuts $t_{\sigma}^{i}$ come in. Namely, for each $i$ and $\sigma$ we insert the whole of $X_{(i)^{\wedge} \sigma}$ into the cut $t_{\sigma}^{i}$, and this then determines the ordering of $Y$. The relation between the various $X_{\sigma} \mathrm{s}$ is given by: $X_{\sigma}$ is contained in the convex hull of $X_{\tau}$ if and only if $\tau$ is a final segment of $\sigma$, and if neither $\sigma, \tau$ is a final segment of the other, then the convex hulls of $X_{\sigma}$ and $X_{\tau}$ are disjoint.

Clearly, $Y$ has cardinality $\kappa$, and is densely ordered without endpoints. The proof that $Y$ is rigid is essentially as before, since the coding of the points of the various $X_{\sigma}$ s has not been 'disturbed', the set of coded points is dense, and the stationary codes, being the final entries of the sequences, are all distinct.

To verify super-density we shall show that $Y$ can be embedded in the set of realizations of each of the cuts $t_{\tau}^{i}$, and as $Y$ is the union of the $X_{\sigma}$ s, we just have to define the embedding on each of these. We map $X_{\sigma}$ into $X_{\sigma^{\wedge}(i)^{\wedge} \tau}$ by $f_{\sigma,(i)^{\wedge} \tau}$. Since the $X_{\sigma^{\prime}} \mathrm{s}$ in which $t_{\tau}^{i}$ is realized are precisely those for which $\sigma^{\prime}$ ends in $(i)^{\wedge} \tau$, this map takes $X_{\sigma}$ into the right set. It remains to show that the embedding is order-preserving. For this, we know that $t_{\sigma}^{j}$ is realized in $X_{(j)^{\wedge} \sigma}$, so we have to show that $f_{\sigma,(i)^{\wedge} \tau} t_{\sigma}^{j}$ is realized in $X_{(j)^{\wedge} \sigma^{\wedge}(i)^{\wedge} \tau}$. But by the choice above, we have $f_{\sigma,(i) \wedge} t_{\sigma}^{j}=t_{\sigma^{\wedge}(i) \wedge}^{j}$, which gives the desired property. This shows that $Y$ is super-dense, and finishes the proof in the case $\kappa$ regular. 
Finally, let us modify the construction to apply to an uncountable singular cardinal $\kappa=\bigcup\left\{\kappa_{\beta}: \beta<\lambda\right\}$ where $\lambda<\kappa$, and we may assume that each $\kappa_{\beta}$ is regular and uncountable. We follow the same steps as in the regular case, adapting where necessary.

In the basic construction of a dense rigid chain of cardinality $\kappa$ we use for $\mathcal{A}$ this time a family of $\kappa$ subsets $S$ of $\kappa$ such that $S$ is a stationary subset of some $\kappa_{\beta}$, and such that if members of $\mathcal{A}$ are stationary subsets of the same $\kappa_{\beta}$, then they are disjoint. (In fact, we could insist that they are all disjoint if we wanted to, by assuming that each $\kappa_{\beta}$ is a successor cardinal, but this is not necessary.) In addition, to make things go smoothly we suppose that all elements of $S \in \mathcal{A}$ have cofinality $\omega$. This is possible since for each $\beta,\left\{\alpha<\kappa_{\beta}: c f(\alpha)=\omega\right\}$ is stationary in $\kappa_{\beta}$ (though not closed unbounded unless $\kappa_{\beta}=\omega_{1}$ ), so by Solovay's result we can start from this set, and take all our stationary sets inside it. This means that we can 'tell' which points in $X=\bigcup_{n \in \omega} X_{n}$ have been coded, and which have not. The 'basic construction' now works as before, noting that now all coded points have uncountable cofinality in the final chain. It will still be true that the set of coded points is dense, and so any non-trivial automorphism $f$ would have to move a coded point $x$. Since the cofinalities of $x$ and $f(x)$ are equal, the stationary sets coding $x$ and $f(x)$ are subsets of the same $\kappa_{\beta}$, and the previous argument can be used.

The remaining parts of the construction ensuring super-density can now be carried out as in the regular case, and this concludes the proof of Theorem 1.2 ,

\section{FURTHER REMARKS}

We describe here how we can modify the examples so that either the chain under consideration, although rigid, becomes non-rigid on removal of a single point, or is non-rigid, and becomes rigid on removal of any single point. The same ideas apply in all cases, so we just do this for dense subsets of the real line, and just for the case of (simple) rigidity. The modification to give many embeddings can be carried out as before.

Suppose then that $(X,<)$ is a dense rigid subset of the real interval $(0,1)$, and suppose further that it is 'locally rigid' in the sense that there is not even any non-identity isomorphism from an open subset of $(X,<)$ to a (possibly different) open subset. We may build from $(X,<)$ a dense non-rigid subset of $\mathbb{R}$ as $Y=$ $\{n+x: n \in \mathbb{Z}, x \in X\}$. Since $X$ is dense in $(0,1), Y$ is dense in $\mathbb{R}$, and $(Y,<)$ is clearly not rigid, since translation by 1 is an automorphism of $Y$. If, on the other hand, we remove any single point $y$ from $Y$, it becomes rigid, since under any automorphism, $y$ will now have to map to an irrational cut in some copy of $X$, which will induce a non-identity local isomorphism, contrary to construction of $X$. The rigid set which becomes non-rigid on removing a (suitable) single point may be taken as $Y \cup\{0\}$ for instance. (There is a difference, however; $Y$ becomes rigid on removing any point, but $Y \cup\{0\}$ only becomes non-rigid on removing the particular point 0.) We further observe that the automorphism group of $(Y,<)$ is infinite cyclic. Presumably modifications of the construction will provide chains with other specified (torsion-free) countable groups as automorphism groups.

Finally, we remark further that it is possible to adapt other constructions to apply to the present context, for instance the ones described in [1] for rigid measurable spaces, though we do not give details. Also, we can construct our rigid super-dense 
subset of the reals so that with respect to the usual field of Borel sets it becomes a strong Blackwell space, as in [2].

\section{REFERENCES}

[1] M. Droste, The existence of rigid measurable spaces, Topology and its Applications 31 (1989), 187-195. MR 90b:28001

[2] M. Droste, Super-rigid families of strongly Blackwell spaces, Proc. Amer. Math. Soc. 103 (1988), 803-808. MR 89e:28006

[3] M. Droste, D. Kuske, R. McKenzie, R. Pöschel, Complementary closed relational clones are not always Krasner clones, Algebra Universalis, to appear.

[4] M. Dugas, R. Göbel: Applications of abelian groups and model theory in algebraic structures, in: 'Infinite Groups' (de Giovanni, Newell, eds.), de Gruyter and Co., Berlin, New York, 1996, 41-62. MR 98m:20030

[5] B. Dushnik and E. W. Miller, Concerning similarity transformations of linearly ordered sets, Bull. Amer. Math. Soc. 46 (1940), 322-326. MR 1:318g

[6] A. M. W. Glass, Ordered Permutation Groups, London Mathematical Society, Lecture Notes, 55, Cambridge University Press, 1981. MR 83j:06004

[7] J. G. Rosenstein, Linear Orderings, Academic Press, 1982. MR 84m:06001

[8] R. M. Solovay, Real-valued measurable cardinals, in D. S. Scott (ed.) Axiomatic set theory, Proc. Symp. Pure Math. XIII Part 1, Amer. Math. Soc. 1971, 397-428. MR 45:55

Institut für Algebra, Technische Universität Dresden, D-01062 Dresden, Germany

E-mail address: droste@math.tu-dresden.de

Department of Pure Mathematics, University of Leeds, Leeds LS2 9JT, England

E-mail address: pmtjkt@leeds.ac.uk 